\title{
Formation and Characterization of Mefenamic acid-Nicotinamide Cocrystal during Co-milling based on X-ray Powder Diffraction Analysis
}

\author{
Dwi Utami ${ }^{1,2^{*}}$, Ilma Nugrahani ${ }^{1}$, Slamet Ibrahim ${ }^{1}$ \\ ${ }^{1}$ School of Pharmacy, Bandung Institute of Technology, West Java, Indonesia. \\ ${ }^{2}$ Ahmad Dahlan University, Yogyakarta, Indonesia.
}

\section{ARTICLE INFO \\ Article history: \\ Received on: 11/05/2016 \\ Revised on: 30/06/2016 \\ Accepted on: 16/08/2016 \\ Available online: 29/10/2016}

Key words:

Cocrystal formation,

mefenamic acid,

nicotinamide, co-milling,

polymorph.

\begin{abstract}
Mefenamic acid (MFA) and Nicotinamide (NIC) cocrystal formation in co-milling treatment was investigated by x-ray powder diffractometry (XRPD). Two polymorphic form of Mefenamic acid (MFA form I and MFA form II) were used to form a cocrystal with nicotinamide. Co-milling treatment was carried out at room temperature in a 1:2 molar ratio of MFA and NIC for various times up to $60 \mathrm{~min}$. Samples were analyzed by XRPD. The XRPD showed that MFA form I and MFA form II formed cocrystal with NIC in the same diffractograms pattern. There was no intermediate amorphous form during milling process. The cocrystal formation mechanism was predicted via intermediate eutectic mixtures. The cocrystal formation from MFA form II (15 min) was faster than from MFA form I (45 min) which may explained by variations unit cell dimensions of MFA form I and MFA form II and also the polymorphic transformation of MFA form I.
\end{abstract}

\section{INTRODUCTION}

Mefenamic acid (MFA) is a well-known as nonsteroidal anti-inflammatory drug (Figure 1) has low aqueous solubility, so its absorption in gastrointestinal tract (GIT) is limited by its dissolution, therefore has a low bioavailability after oral administration (Merck Index, 1996). Many approaches have been studied to enhance solubility and dissolution profile of MFA. The techniques such as solid dispersion with polyvinylpyrrolidone (Kang et al, 2015) or modified starch (Vikram, 2012), a complex with cyclodextrin (Hladon et al, 1999), and also a cocrystal formation (Fabian et al, 2011) have been tried. The progress of solid stage of MFA in various pharmaceutical dosage form was also hindered by polymorphic transformation of MFA along of the formulation process.

* Corresponding Author

Email: utamipurwantoro @ yahoo.com
MFA have been reported three polymorphic form : MFA form I (Mc. Connell, 1976), MFA form II (Lee et al, 2006) and form III (SeethaLekshmi and Row, 2013). The MFA form I is stable at room temperature and has a melting point $170{ }^{\circ} \mathrm{C}$ and $232^{\circ} \mathrm{C}$. The MFA form I is a commercially marketable form of Mefenamic acid. The MFA form II is metastable ones and has a melting point $232{ }^{\circ} \mathrm{C}$, on the other hand, is stable at higher temperature ((SeethaLekshmi and Row, 2013). The MFA form II has also been investigated widely due to its advantage than MFA form I in solubility and dissolution rate (Dixit et al, 2012; Romero et al, 1999). The polymorphic transformation of MFA I to MFA II to be formed by co-milling (Kato et al, 2014), cryomilling process (Kang et al, 2015), heating crystals of MFA I above the transition temperature $\left(165-175^{\circ} \mathrm{C}\right)$ and by rapid cooling of a supersaturated of MFA I in DMF (Romero et al, 1999 ; Surov et al, 2009) Fabian et al (2011) has been studied cocrystal formation of five fenamic acid drugs, including MFA with NIC based on supramolecular heterosynthon of both. The cocrystal of MFA be formed by comilling and liquid assisted co-milling. 
The MFA-NIC cocrystal could only be prepared as polycrystalline powder. The single crystal of MFA-NIC cocrystal not have been found yet, and the packing of cocrystal was determined by powder x-ray data. The investigation of MFA cocrystal techniques are still poses a challenges, despite its defined structure and its psychochemical properties. Due to the difficulties in achieving the single crystal of MFA-NIC by solvent evaporation, the investigation of cocrystal formation by milling process is interesting to be explored. The knowledge of kinetic formation is useful to investigate the pathway of cocrystal formation, especially in the drugs such as MFA that the polymorphic transformation caused by milling process. It should be possible that the cocrystal formation is built by polymorphic transformation of drug.

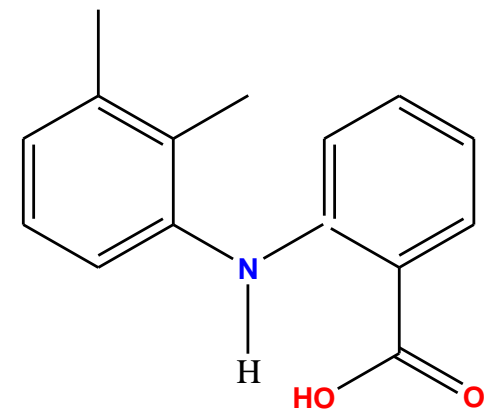

Mefenamic acid (MFA)

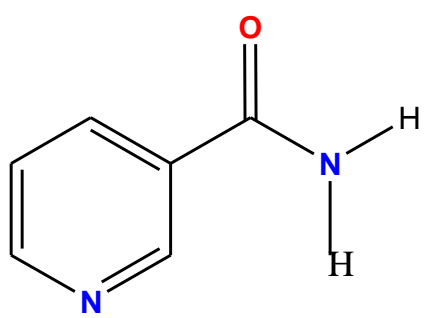

Nicotinamide (NIC)

Fig. 1: The chemical structure of mefenamic acid (MFA) and micotinamide (NIC)

The usage of different polymorphic forms may lead increasing chance for cocrystal formation, because polymorphism is based on molecular flexibility. The different crystal lattice arrangement with structurally less rigid with another molecules could be easier to be packed (Aakeröy, 1997).

For example, the carbamazepine-nicotinamide cocrystal was qualitatively found to be faster for carbamazepine dihydrate, allowed by carbamazepine form I and carbamazepine form III, although carbamazepine form III was the most stable at ambient temperature (Cheing et al, 2009). It will be preferable in industrial scale to use the substance that has the less time production. In the field of kinetic cocrystal formation study, some of analytical method has been used in monitoring and characterizing the solid state transformations of drugs. KorangYeboah et al, 2016, have been used NIR spectrometry, Raman spectrometry, solid state NMR and also powder x-ray diffraction in the solid state stability of theophylline. In this study, XRPD was used due to its selectivity and simplicity to detect the solid state transformation.

The appearance of characteristic peaks of cocrystal could be indicate the initial cocrystal formation. Rehder et al (2011) reported that upon milling, the significant changes in the XRPD pattern in piracetam-tartaric acid/citric acid can be detected. Chieng et al (2009) was also found that the evolution of carbamazepine - nicotinamide cocrystal peaks upon milling is clearly visible by increasing comilling time.

The aim of this study was to investigate the formation of MFA-NIC cocrystals by milling, using MFA form I and MFA form II and NIC as starting material. The cocrystal formation study was investigated as a function of the milling and the polymorphic form by using XRPD. The relevant implication of the findings presented here is useful for the cocrystal researchers to gain a deeper understanding of the polymorphic forms compounds in the cocrystal formation.

While for those who are not involved in the cocrystal exploration, is to anticipate the possibility of the formation of cocrystal for pharmaceutical processes, such as grinding, especially for active ingredients that susceptible to the polymorph transformation by grinding process.

\section{MATERIAL AND METHODS}

\section{Materials}

Mefenamic acid (2-(2, 3-dimethylphenyl) amino benzoic acid) $(99.0 \%$ Pyridam), Nicotinamide (99.0 \%, Sigma), Dimethylformamide (>99.5\%, E-Merck) and used without further purification for crystallization of form II of mefenamic acid.

\section{Methods}

\section{Preparation of Mefenamic Acid form II}

Form II of MFA were prepared in N,N-dimethylformamide solution. An amount of MFA form I were dissolved in N,N-di-methylformamide and stir at 600 RPM in $40{ }^{\circ} \mathrm{C}$ up to give a clear solution. The clear solution was cooled to $-12{ }^{\circ} \mathrm{C}$ in frezee refrigerator. The solution was maintained at this temperature until most of the mefenamic acid was crystallized. The crystalls were filtered and dried at room temperature. Then the dried crystals were dried at $70^{\circ} \mathrm{C}$ to obtained mefenamic acid form II.

All of the crystal were characterized by Fourier transform Infrared (FTIR), Differential Scanning Calorimetry (DSC), X-ray Powder diffraction (XRPD) and Scanning Electron Microscopy (SEM). 


\section{Physical mixing and co-milling}

Physical mixtures were obtained by gentle mixing of MFA and NIC in a 1:2 molar ratio in a glass mortar with a glass pestle for $1 \mathrm{~min}$. Dry co-milling was performed by co-milling MFA with NIC at a 1:2 molar ratio in automatic grinder (Retsch mortar RM 100) at 5 scale. Milling was carried our for predefined time periods from $15 \mathrm{~min}$ to $60 \mathrm{~min}$. The samples were used to investigate the kinetic of cocrystal formation by XRPD analysis.

\section{Characterization methods}

\section{Fourier Transform Infrared Spectroscopy (FTIR)}

Fourier transform infrared measurements were conducted using an Infrared Spectrophotometer (8400S, Shimadzu) and $\mathrm{KBr}$ beam splitter. The range was set from 350 to $4000 \mathrm{~cm}^{-1}$ with a 4 $\mathrm{cm}^{-1}$ resolution.

\section{Differential Scanning Calorimetry (DSC)}

The samples were weighed (1-5 mg) into a Differential Scanning Calorimetry (Linseis sta pt 1600 and Q-20 Thermal Analysis). TA instruments standard aluminium pan using a micro balance and tweezers. The pan was covered with a lid and crimped using a TA crimper.

The reference pan was crimped similiar to the sample pans, but without any substance. Thermograms were recorded under a constant nitrogen gas flow of $50 \mathrm{~mL} / \mathrm{min}$. The DSC apparatus was calibrated with regard to temperature and enthalpy using indium as a standard. The heating was set to $10 \mathrm{~K} / \mathrm{min}$ in a range from 30 to $250^{\circ} \mathrm{C}$.

\section{$X$-ray Powder Diffractometry (XRPD)}

The X-ray powder diffraction analysis was performed in Philips PW 1710 BASED and illuminated with $\mathrm{Cu}-\mathrm{K} \alpha$ radiation $(\lambda=1.5418)$ at a tube voltage of $40 \mathrm{kV}$ and a tube current of $35 \mathrm{~mA}$. The samples were analysed over a $2 \theta$ range of $5-45^{\circ}$ with increment of $0.019^{\circ}$ at a rate of $7 / \mathrm{min}$.

\section{Scanning Electron Microscopy (SEM)}

The samples were stuck on the stubs using carbon adhesive tape and coated with gold. SEM images were obtained with table-top scanning electron microscope (JEOL JSM-6360LA) at an acceleration voltage of $15 \mathrm{kV}$. The SEM was equipped with a solid-state backscatter detector and operated in a standard imaging mode for obtaining the images.

\section{RESULT AND DISCUSSION}

\section{Characterization of MFA form I and MFA form II}

The MFA form I was used as received without recrystalized. The MFA form II were recrystalized from DMF as described by Kang et al (2015). The IR absorption bands (Fig. 2) in the FTIR spectrum of MFA form I and MFA II showed characteristic differences in the region of wave number $3345 \mathrm{~cm}^{-1}$ as NH stretching in MFA form II and $3306 \mathrm{~cm}^{-1}$ in MFA form I. The $-\mathrm{OH}$ group bending and vibration of $\mathrm{COOH}$ showed at 1245 $\mathrm{cm}^{-1}$ in MFA form II and $1253 \mathrm{~cm}^{-1}$ in MFA form I. The $\mathrm{C}=\mathrm{O}$ stretching at $1567 \mathrm{~cm}^{-1}$ of MFA form II and $1573 \mathrm{~cm}^{-1}$ of MFA form I. All FTIR absorption profiles of MFA form I and form II were matched the FTIR profiles that reported before (Dixit et al, 2012 ; Kang et al, 2015). The brief vibration values of each chemical as shown in Table 1. The specific characteristics of MFA form 1 and form 2 were also shown from thermogram profile. The thermogram showed an endothermic peak at $168.9^{\circ} \mathrm{C}$ and $230.1{ }^{\circ} \mathrm{C}$ in MFA form I due to the transformation to form II, and MFA form II exhibited only an endothermic peak at $229.9^{\circ} \mathrm{C}$. Figure 3 showed the thermogram from DSC scanning.

Table 1. Wavenumber of functional groups of MFA form I and MFA form II by DMF recrystallization $\left(\mathrm{cm}^{-1}\right)$.

\begin{tabular}{|c|c|c|c|c|}
\hline Functional group & 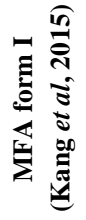 & 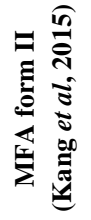 & 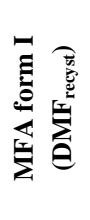 & 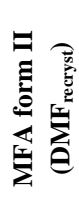 \\
\hline $\mathrm{N}-\mathrm{H}$ bending & 3311 & 3347 & 3305 & 3347 \\
\hline $\mathrm{C}=\mathrm{O}$ bending & 1650 & 1648 & 1650 & 1650 \\
\hline $\mathrm{N}-\mathrm{H}$ stretching & 1577 & 1567 & 1573 & 1569 \\
\hline Benzene ring stretching & 1511 & 1496 & 1508 & 1508 \\
\hline Vibration of $\mathrm{COOH}$ & 1259 & 1249 & 1253 & 1245 \\
\hline $\mathrm{CH}_{3}$ rocking vibrations & 892 & 916 & 887 & 918 \\
\hline $\mathrm{CH}$ out-of-plane bending vibration & 755 & 744 & 752 & 740 \\
\hline
\end{tabular}

Table 2. DSC parameters of MFA form I, MFA form II, Nicotinamide, and comilling of MFA form I/form II-NIC.

\begin{tabular}{lcc}
\hline Samples & Melting point $\left({ }^{\circ} \mathbf{C}\right)$ & $\mathbf{\Delta H}(\mathbf{J} / \mathbf{g})$ \\
\hline MFA form I & 169,9 & $-7,74$ \\
& 230,1 & $-121,22$ \\
MFA form II & 229,9 & $-139,30$ \\
Nicotinamide & 129,6 & $-221,71$ \\
MFA form I-NIC co-grinding & 123,5 & $-50,98$ \\
MFA form II-NCIC co-milling & 124,1 & $-49,12$ \\
\hline
\end{tabular}

As stated by SeethaLaksmi and Row (2012), the MFA form I have different crystal lattice with MFA form II. The different of MFA form I and MFA form II due to its molecular packing can be detected by X-ray diffraction analysis. The MFA form I showed specific diffractogram at $2 \theta: 6.4^{\circ}, 21.35^{\circ}$ and $26.21^{\circ}$, while MFA form II showed specific diffractogram at $2 \theta$ : $11.73^{\circ}, 17.86^{\circ}, 24.73^{\circ}$ and $25.49^{\circ}$

Figure 3 showed the diffractogram of MFA I and MFA II. Based on FTIR absorption band, thermogram of DSC scanning and diffractogram pattern, can be concluded that MFA form I and MFA form II that have been produced were matched with the results that previous before. Figure 4 represents the surface morphology of MFA form I and MFA form II. The MFA form I crystals were stick particles and smaller size than MFA form II, whether the MFA form II crystals were in cube-shaped particles. 

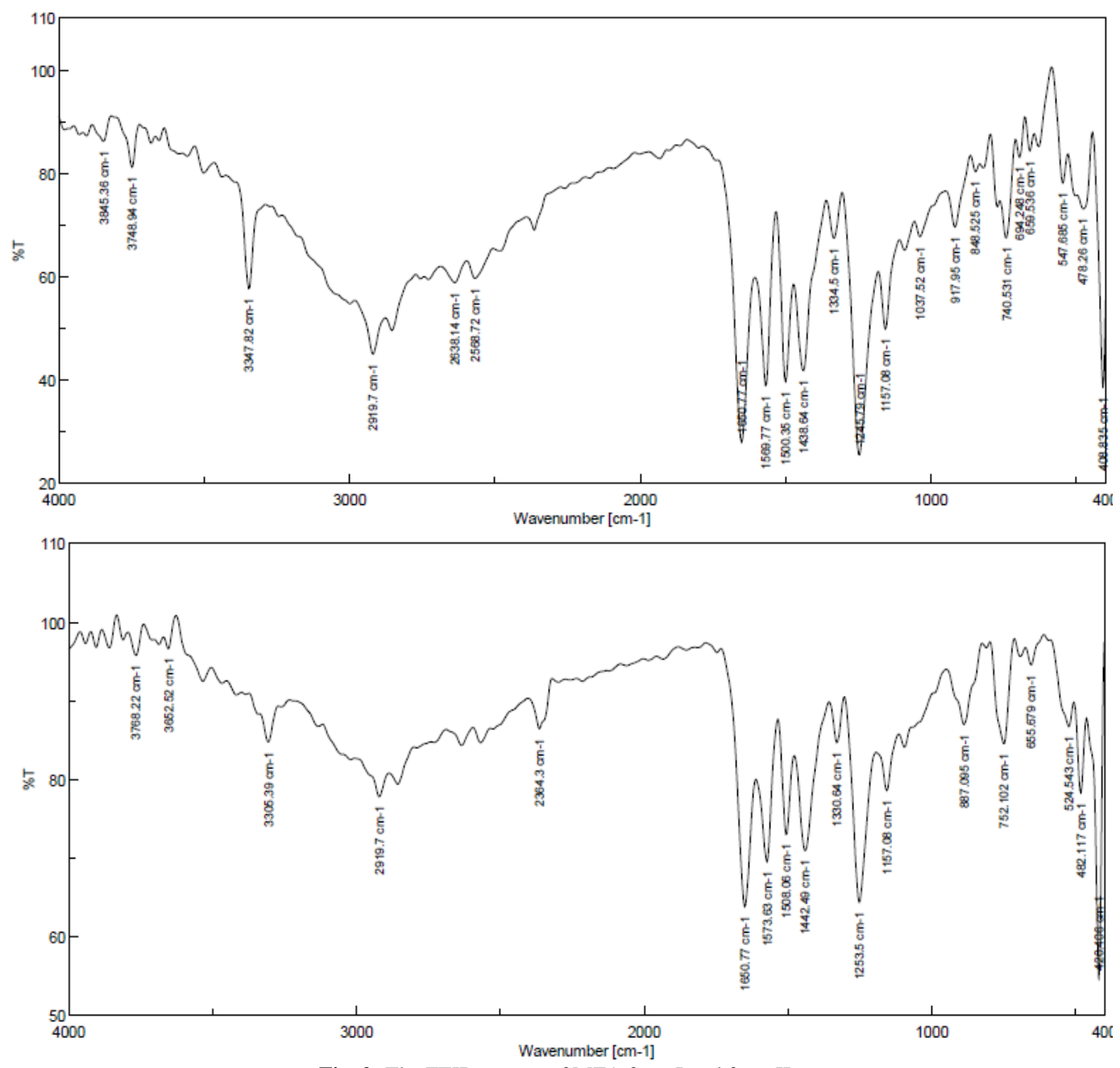

Fig. 2: The FTIR spectra of MFA form I and form II.
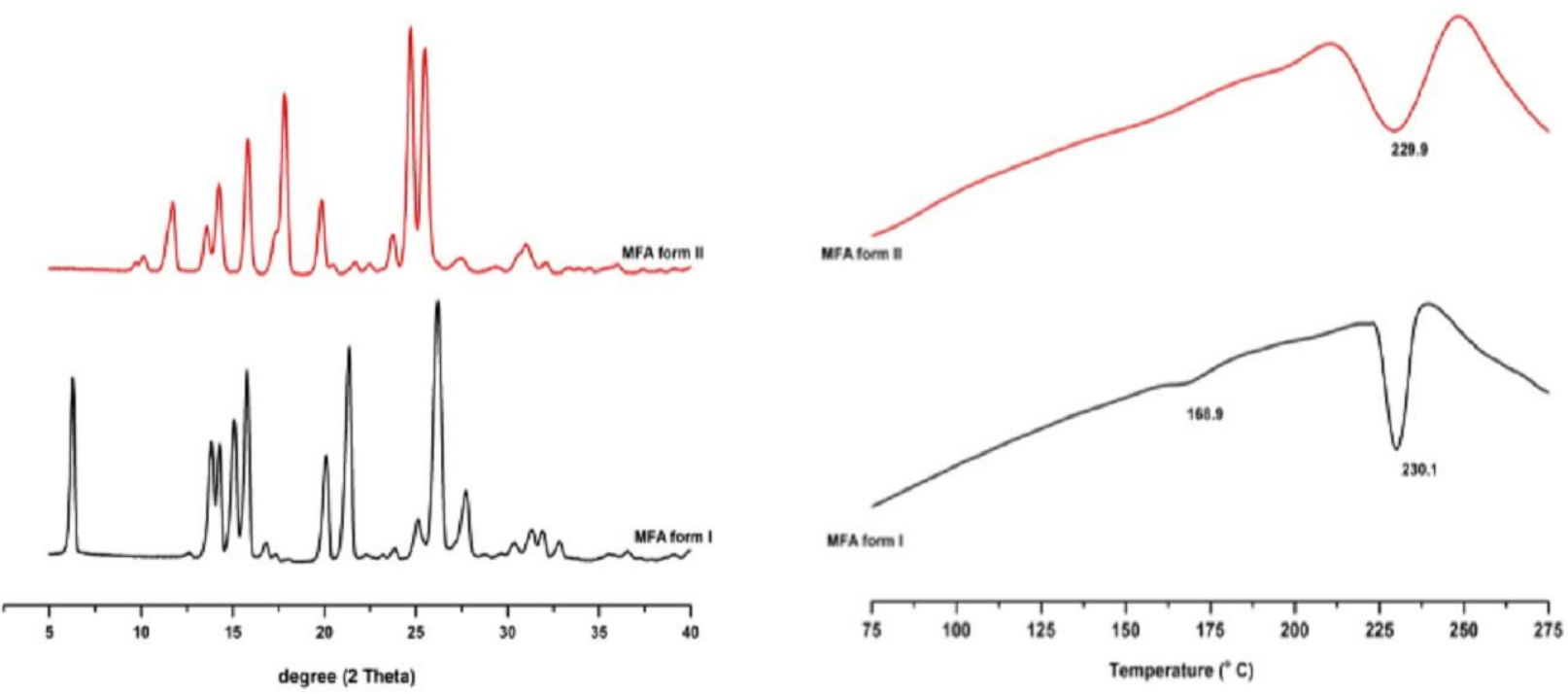

Fig. 3: The X-ray Powder diffraction patterns and DSC profiles of MFA form I and II. 


\section{Formation of MFA-NIC Cocrystal from polymorph form I and form II by Co-Milling}

In the investigation of kinetic formation of MFA-NIC cocrystal, the co-milling of MFA-NIC was used MFA form I, MFA form II and NIC form II as starting material. Figure 5 showed the diffractograms of the MFA form I and MFA form II co-milled with NIC for up to $60 \mathrm{~min}$. The diffractograms of MFA form I, MFA form II, NIC and their respective physical mixtures were also shown for comparation. The crystal lattice spacing changes will cause the changes of diffractogram pattern, which characterized by the appearance or loss of the peaks (Nugrahani et $a l, 2015)$. According to Fabian et al, 2011, the co-crystal of MFA and NIC will form the new peaks with moderate intensity at $2 \theta$ : $7.2^{\circ} ; 9.3^{\circ} ; 12.1^{\circ}$ and two peaks with high intensity around $15.4^{\circ}$. Diffractogram data in fig. 4 showed that cocrystal resulted from MFA form I and form II provide the same peaks. Both of MFA form I and MFA form II led the formation of the of the same cocrystal. This result is in line with the formation of carbamazepin-nicotinamide cocrystal (Chieng et al, 2009) and piracetam-citric acid/tartaric acid cocrystal (Rehder et al, 2011).

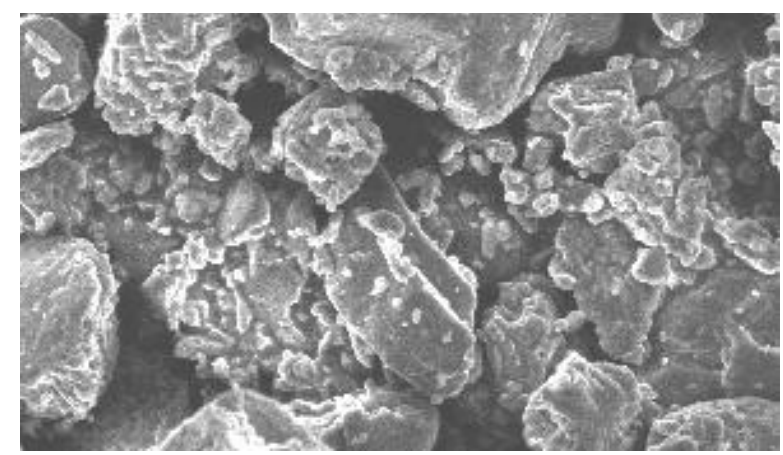

A

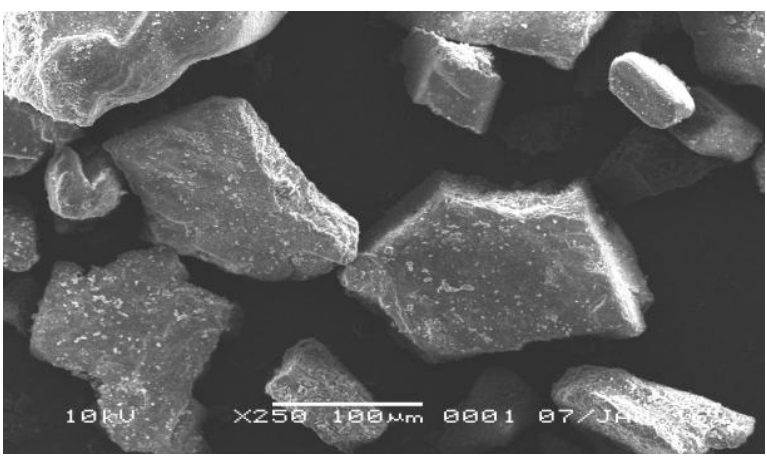

B

Fig. 4: The SEM photographs of MFA form I (a) and form II (b)

When MFA form I was used, the diffractograms of samples milled for 15 minutes was similar to those physical mixtures, that mostly of characteristic peaks of MFA form I and NIC. The peak intensity of MFA form I and NIC were reduced during milling process. The reduced intensity of characteristic diffractogram of MFA form I was clearly recognised at $2 \theta: 6.2^{\circ}$. The intensity of this peak was reduced gradually during the milling process. After 60 minutes milling process the peak was still appear with low intensity $(32.3 \%)$. The diffractogram pattern also showed that the peak intensity in both MFA form I-NIC and MFA form II-NIC cocrystal formation process were sharp and not identifiable some broader peaks with lower intensity. Meanwhile the intermediate amorphous state was not observed in this study.
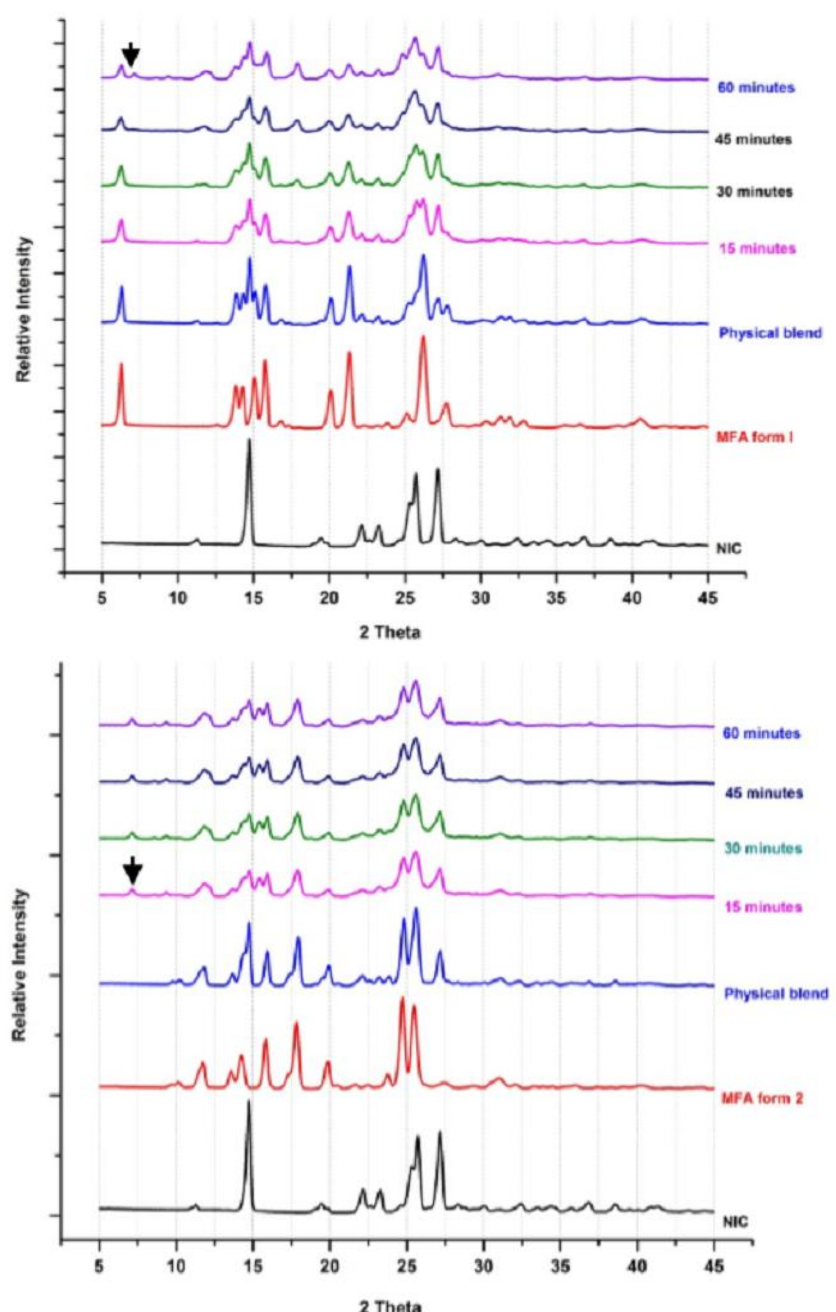

Fig. 5: The kinetic formation of MFA form I cocrystal and MFA form II cocrystal based on diffractograms changes

According to Friscic and Jones (2008) there are three mechanism possibility in cocrystal formation by dry-grinding : molecular diffusion, intermediate formation of eutectic mixtures, and intermediate formation of an amorphous phase. In the MFANIC cocrystal formation study, the absence of amorphous phase during millimg process indicated that cocrystal mechanism of its was not via amorphous phase. The possibility of cocrystal formation mechanism was via intermediate of an eutectic mixtures rather than molecular diffusion. In molecular diffusion mechanism, one or both reactants must exhibit significant vapor pressures in the solid state (Friscic and Jones, 2008). In the other state, the reactant must be a volatile solid, whether MFA and NIC were not volatile compounds. The eutetic mixtures was indicated 
by lower melting point of solid ground than each component (Cherukuvada and Row, 2014). The melting point of physical mixtures and solid ground of MFA form I-NIC and MFA form INIC was around $121-122^{\circ} \mathrm{C}$, lower than melting point of MFA form I $\left(168.9^{\circ} \mathrm{C}\right.$ and $\left.230.1^{\circ} \mathrm{C}\right)$, MFA form II $\left(229.9^{\circ} \mathrm{C}\right)$ and NIC $\left(129^{\circ} \mathrm{C}\right)$. However, the mechanism of MFA-NIC cocrystal formation by grinding process was not completely understood and warrant further investigation. The other experiment design study of MFA-NIC cocrystal formation via eutetic mixtures need to be explored.

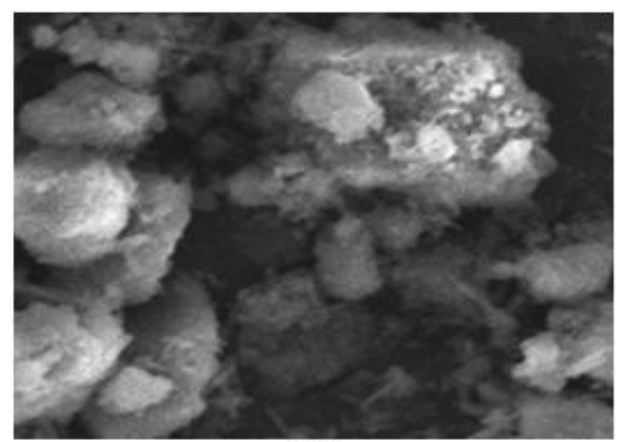

(a)

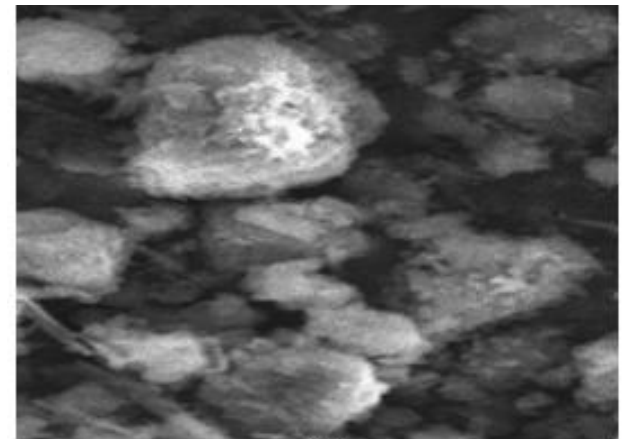

(b)

Fig. 6: The SEM photographs of co-grinding of MFA form I (a) and cogrinding of MFA form II-NIC (b)

Interestingly, the rate of cocrystal formation was found to be different for the two MFA polymorphs. Based on XRPD results, MFA-NIC cocrystal formation was faster when MFA form II was used. In the XRPD pattern in 15 minutes of milling, the initial of diffractogram of MFA-NIC cocrystal already be observed at $2 \theta: 7.18^{\circ}$ and $15.4^{\circ}$ (Figure 4B; 15 minutes, arrow). While the same peak was observed in MFA form I-NIC after milling process for 45 minutes (Figure 4A; 45 minutes, arrow). When the milling time was extended up to 60 minutes, the characteristic diffractogram of MFA-NIC cocrystal was present in both MFA form I and form II.

The variation in the rate of cocrystal formation could be explained by the differences in the crystal packing of MFA I and MFA II. Although both polymorph were crystallized in the triclinic space group, the significant variations in the unit cell dimensions has been observed, especially the hydrogen bonds of the carboxylic acid that makes a symmetric $\mathrm{O}-\mathrm{H}---\mathrm{O}$ synthon and additional $\mathrm{C}-\mathrm{H}$ $--\pi$ and $\pi---\pi$ interactions. The type of dimer interaction in MFA I and MFA II were similiar, but based on the Hirshfield surface (HS) analysis, the contribution of each type interaction to the structure stabilization is varies. The contribution of $\pi---\pi$ interactions in the MFA form II is $100 \%$ more than MFA I. The possible $\pi---\pi$ stacking in MFA I is less than MFA II and the C-H$--\pi$ is more to stabilizing the dimer. Therefore MFA I is more stable than MFA form II in the homodimer form (SeethaLekshmi and Row, 2012). In the crystal lattice point of view, the synthon interaction of MFA form II with NIC was faster than MFA form I due to the intramolecular hydrogen bond of MFA form II was breakout easily than MFA form I.

The second explanation, the result also showed that during grinding process, in the 30 minutes of MFA form I-NIC comilling there was detected the diffractograms of MFA form II (at $2 \theta: 11.7^{\circ}$ and $17.9^{\circ}$ ) that not present in the physical mixture. This finding indicated that during milling process, MFA form I was transformed to MFA form II and in line with research that have been reported before. Milling process (grinding and cryomilling) can induce the transformation of MFA form I to MFA form II (Kato et al, 2014; Kang et al, 2015). The polymorphic transformation during milling process was a usual phenomenon in pharmaceutical substances. Lin et al, 2010, reported the polymorph transformation of gabapentin (anticonvulsant agent) during the $120 \mathrm{~min}$ milling process both in the absence and presence of additive. It seems that the energy that given by grinding process was also used to polymorphic transformation of MFA form I. The energy that obtained from milling process was not only used to interact with the NIC but also to transform the MFA form II. As the final result, the cocrystal formation with NIC of MFA form I was slower than the MFA form II.

Furthermore, as the summary, this study was demonstrated that MFA-NIC cocrystal formation can be achieved by co-milling of MFA form or MFA form II and has the same diffractogram pattern. There was no amorphous phase intermediate during milling process and the cocrystal formation mechanism was predicted via intermediate eutectic mixtures. The rate of cocrystal formation was faster in MFA form II than MFA form I. The significant variations unit cell dimensions of MFA form I and MFA form II and also the polymorphic transformation of MFA form I were predicted to be influenced to the rate of cocrystal formation.

Finally, the study of cocrystal formation drugs in various polymorphic form is still important to be explored, in order to obtain the deep understanding cocrystal formation in different molecular packing crystal. And also find the suitable polymorphic form to be formed as cocrystal that has the optimal physicochemical properties such as the rate of formation, stability, hygrospicity, and the other aspect in manufacturing process.

\section{CONCLUSION}

The result of this study showed that MFA-NIC cocrystal can be prepared by co-milling from both MFA polymorph (form I and form II) with the same diffractogram pattern. The rate of 
cocrystal formation in MFA form II was faster than MFA form I. The absence of amorphous phase during milling process indicated that cocrystal formation mechanism was not via intermediate amorphous phase.

\section{ACKNOWLEDGMENT}

The authors are thanksful to Pyridam Farma pharmaceutical, Indonesia for the Mefenamic acid drug.

\section{Financial support and sponsorship: Nil.}

Conflict of Interests: There are no conflicts of interest.

\section{REFERENCES}

Aakeröy, C.B., Crystal engineering : strategies and architectures. Acta Crystallogr. B, 1997, 53 : 569-586.

Aakeröy, C.B, Beatty. A.M; Helfrich. B.A; Nieuwenhuyzen. M, Do Polymorphic Compounds Make Good Cocrystallizing Agents ? A Structural Case Study that Demonstrates the Importance of Synthon Flexibility, Crystal Growth and Design, 2003;1:159-165.

Chieng N.; Hubert, M.;Saville, D.; Rades, T.; Aaltonen, J., Formation Kinetics and Stability of Carbamazepine-Nicotinamide Cocrystal Prepared by Mechanical Activation, Crystal Growth and Design, 2009;9:2377-2386.

Cherukuvada. $S$ and Row. T.N.G, Comprehending the Formation of Eutectic and Cocrystals in Terms of Design and Their Structural Interrelationships, Crystal Growth and Design, 2014;14:41874198

Dixit, M.; Kini, A.G.; Kulkarni, P.K., Enhancing The Dissolution of Polymorphs I and II of Mefenamic Acid by Spray Drying, Turki. Journal of Pharmaceutical Sciences, 2012;9:13-26.

Fabian, L.; Hamill, N.; Eccles, K.S.; Moynihan.R.; McCausland, L.; Lawrence, S.E.; Cocrystal of Fenamic Acids with Nicotinamide, Crystal Growth and Design, 2011;11:3522-3528.

Friscic Tomislav and Jones William, , Recent Advances in Understanding the Mechanism of Cocrystal Formation via Grinding, Crystal Growth and Design, 2009;9:1621-1637.

Hladon T, Pawlaczyk J, Szafran BJ, Stability of Mefenamic acid in the Inclusion Complex with $\beta$-cyclodextrin in The Solid Phase. Incl Phenom Macro, 1999;35:497-506.

Kang, N.; Lee, J.; Choi, J.N.; Mao, C.; Lee, E.H.; Cryomilling-induced Solid Dispersion of Poor Glass Forming/Poorly Water-Soluble Mefenamic Acid with Polyvinylpyrrolidone K12, Drug Development and Industrial Pharmacy, 2015;41:978-988.
Kato, F.; Matsuda, Y.; Watano, S.,Effect of Co-milling on Solid State Stability of Mefenamic Acid Polymorphs, Japan. Sociate.Powder. Technology, 2014;51: 678-685.

Korang-Yeboah M.; Rahman Z.; Shah D., Mohammad A.; Wu S.; Siddiqui A; Khan M.A; Impact of formulation and process variables on solid-state stability of theophylline in controlled release formulations, International Journal of Pharmaceutics, 2016 ; 499 : 20-28.

Lee EH, Byrn SR, Carvajal MT, Additive-Induced Metastable Single Crystal of Mefenamic Acid, Pharm res, 2006;10:2375-2380.

Lin Shan-Yang, Hsu Cheng-Hung, Ke Wen-Ting, Solid-state transformation of different gabapentin polymorphs upon milling and comilling, International Journal Of Pharmaceutics, 2010; $396: 83-90$.

McConnell JF, Company FZ. N-(2,3-xylyl) anthranilic acid, $\mathrm{C}_{15} \mathrm{H}_{15} \mathrm{NO}_{2}$ mefenamic acid, Cryst Struct Commun, 1976;5:861-864.

Nugrahani. I, Pertiwi. E.A, Putra O.D, Theophyllline-NaSaccharrine Single Crystal Isolation For Its Structure Determination, International Journal of Pharmacy and Pharmaceutical science, 2015;7:1524.

Rehder, S.; Klukkert, M.; Lobmann, K.A.M.; Strachan, C.J.; Sakmann, A.; Gordon, K.; Rades, T.; Leopold, C.S., , Investigation of The Formation Process of Two Piracetam Cocrystal during Co-milling, Pharmaceutics, 2011;3:706-722.

Romero S, Escalera B, Bustamante P, Solubility Behavior of Polymorphs I and II of Mefenamic acid in Solvent Mixtures, Int J Pharm, 1999;178: 193-202.

SeethaLekshmi, S.; Row, T.N.G., Conformational Polymorphism in a Non-steroidal Anti-inflammatory Drug, Mefenamic Acid, Crystal Growth and Design, 2012;12:4283-4289.

Surov A.O, Terekhova, I.V, Bauer-Brandl, A., Perlovich, G.L., Thermodynamic and Structural Aspects of Some Fenamate Molecular Crystals, Cryst. Growth. Des, 2009;9:3265-3272.

Vikram A, Firoz S, Kishore D, et al, Enhancement Solubility and Dissolution of Mefenamic acid by Modified Starch, Int. J. Pharm Sci Tech, 2012;7:85-92.

\section{How to cite this article:}

Utami D, Nugrahani I, Ibrahim S. Formation and Characterization of Mefenamic acid-Nicotinamide Cocrystal during Co-milling based on X-ray Powder Diffraction Analysis. J App Pharm Sci, 2016; 6 (10): 075-081. 